\title{
High efficiency D-A structured luminogen with aggregation-induced emission and mechanochromic characteristics
}

\author{
LU YaWei ${ }^{1}$, TAN YeQiang ${ }^{2}$, GONG Yong Yang ${ }^{1}$, LI Hong $^{1}$, YUAN WangZhang ${ }^{1 *}$, \\ ZHANG YongMing $^{1 *} \&$ TANG Ben Zhong ${ }^{3 *}$ \\ ${ }^{1}$ School of Chemistry and Chemical Engineering, Shanghai Jiao Tong University, Shanghai 200240, China; \\ ${ }^{2}$ Department of Polymer Science and Engineering, Zhejiang University, Hangzhou 310027, China; \\ ${ }^{3}$ Department of Chemistry, The Hong Kong University of Science \& Technology, Clear Water Bay, Kowloon, Hong Kong, China
}

Received December 18, 2012; accepted March 21, 2013; published online June 6, 2013

\begin{abstract}
A novel luminogen, CZ2TPAN, with typical D-A architecture was obtained. It shows intramolecular charge transfer and aggregation-induced emission characteristics with high solid-state efficiency of $65.3 \%$. Moreover, it exhibits reversible mechanochromic behavior between crystalline and amorphous states with remarkable emission color change from green (504 nm) to yellow (545 nm).
\end{abstract}

aggregation-induced emission, intramolecular charge transfer, mechanochromic

Citation: $\quad$ Lu Y W, Tan Y Q, Gong Y Y, et al. High efficiency D-A structured luminogen with aggregation-induced emission and mechanochromic characteristics. Chin Sci Bull, 2013, 58: 2719-2722, doi: 10.1007/s11434-013-5893-0

Mechanofluorochromism is a change in fluorescence color induced by mechanical stimuli, accompanied with a reversion to the original emission color by heating, recrystallization, or exposure to solvent vapors [1-3]. Solid emitters showing mechanofluorochromism are attracting increasing interests due to their fundamental importance and potential applications in optical storage, chemical and stress sensors, security papers, optoelectronic devices [4-8], etc. One effective way to tune or switch the luminescence of organic solids is to control the molecular packing [9]. It has been generally believed that the fluorescent color change is attributed to the interconversion between two different molecular packing modes [10]. However, it is still difficult to achieve efficient mechanochromic luminophores with high contrast since most traditional luminogens suffer from thorny aggregation-caused quenching (ACQ) problem [11]. So far, only limited organic mechanofluorochromic materials have been reported and the precise mechanism and design principle are not yet clear [12]. Therefore, it is of significant importance to explore new mechanochromic lu- minogens, particularly those with high solid-state efficiency and remarkable contrast.

Contrary to the ACQ dyes, in 2001, we reported a novel kind of luminescent materials exhibiting aggregationinduced emission (AIE) characteristics, which faintly or nonemissive in solutions but become strong emitters when aggregated [13-17]. The discovery of AIE phenomenon affords great potential for the creation of novel mechanochromic luminogens. Fluorophores with combined advantages of both mechanochromic and AIE-active molecules would have a wide range of potential applications. Herein, we report our recent work on the preparation of such kind of materials with electron donor-acceptor (D-A) architecture. The resulting compound CZ2TPAN (Scheme 1) shows typical intramolecular charge transfer (ICT) and AIE characteristics, and moreover high solid-state efficiency as well as distinct mechanochromism.

The target compound CZ2TPAN was obtained in moderate yield $(56 \%)$ according to the synthetic route shown in Scheme 1. Briefly, nucleophilic substitution reaction be-

*Corresponding authors (email: wzhyuan@sjtu.edu.cn; ymzsjtu@yahoo.com.cn; tangbenz@ust.hk) 
tween 3,6-dibromocarbazole (1) and bromoethane (2) generated 3,6-dibromo-9-ethylcarbazole (3), then coupled with 2-(4-pinacolatoboronphenyl)-3,3-diphenylacrylonitrile (4) [18] through (dppf) $\mathrm{PdCl}_{2}$ catalyzed Suzuki coupling, producing the resulting CZ2TPAN in $56 \%$ yield. The intermediates and final products were fully characterized by ${ }^{1} \mathrm{H}$ NMR, ${ }^{13} \mathrm{C}$ NMR, and mass spectrum (Figure S1), with satisfactory results obtained (Supporting Information).

CZ2TPAN consists of typical electron-donating carbazole and electron-accepting cyano groups. Such D-A structure could form interesting ICT process in CZ2TPAN. As depicted in Figure 1(a), CZ2TPAN exhibits two characteristic absorption bands in varying solvents, giving shorter/longer-wavelength absorptions of $296 / 359 \mathrm{~nm}$. While the former is ascribed to the $\pi-\pi^{*}$ transitions, the latter is attributable to the ICT transition between electron rich carbazole and electron deficient cyano segments.

Normalized emission profiles (Figure 1(b)) of CZ2TPAN in varying solvents are quite different owing to the solvent polarity dependent ICT processes. In non-polar to moderately polar solvents like $n$-hexane, toluene and THF, the emissions mainly come from the locally excited (LE) states with emission maxima at 402 ( $n$-hexane), 413/431 (toluene) and $421 / 530 \mathrm{~nm}$ (THF). With increasing solvent polarity, the ICT state emissions become predominant, whose emission peaks are 541 and $581 \mathrm{~nm}$ in DCM and DMF, respectively, exhibiting obvious bathochromic shift. Meanwhile, LE state

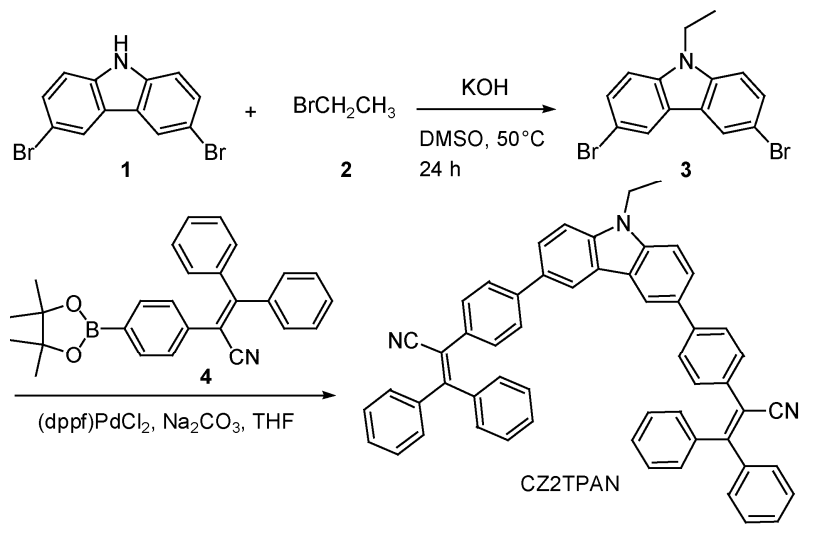

Scheme 1 Synthetic route to CZ2TPAN.
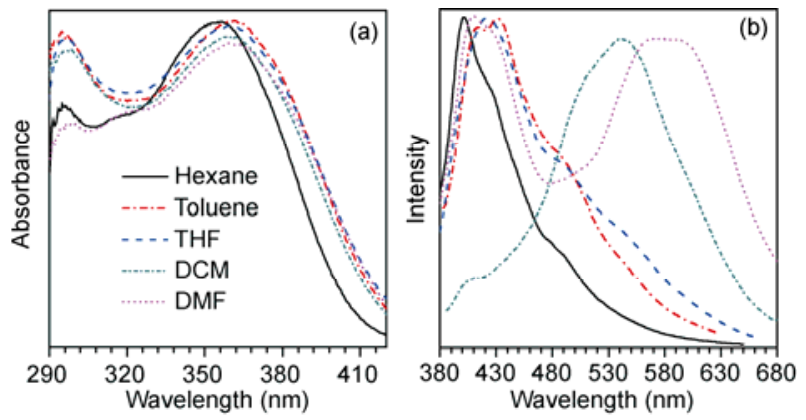

Figure 1 Normalized (a) absorption and (b) emission spectra of CZ2TPAN in varying solvents. Concentration $=20 \mu \mathrm{mol} / \mathrm{L}$; excitation wavelength $=$ $360 \mathrm{~nm}$. emissions at 408 (shoulder) and $410 \mathrm{~nm}$ (peak) are also observed in DCM and DMF, respectively. It is noted that the relatively larger fraction of LE emission in DMF than in DCM might indicate that the ICT emission is more effectively quenched compared to LE emission in DMF, thus giving rather weak but comparable LE and ICT emissions.

CZ2TPAN readily dissolves in common organic solvents such as DMF and THF but is insoluble in water. When illuminated under UV light, no emission was seen from its solutions, whereas intense fluorescence from the solid powders was observed, indicating that aggregation has induced its light emission. The AIE-active nature was further confirmed by the photoluminescence (PL) spectra measurement. As shown in Figure 2, rather weak PL intensity with almost a flat line is obtained in THF, and even when the water fraction $\left(f_{\mathrm{w}}\right)$ increases to $80 \%$, only a feeble signal is recorded due to its genuine dissolution. However, when $f_{\mathrm{w}}>85 \%$, addition of even a small amount of water causes dramatic enhancement in PL intensity, which should be ascribed to the formation of nanosuspensions owing to the deterioration of solvating power of the THF/water mixture. At a $f_{\mathrm{w}}$ of $90 \%$, the PL intensity at $541 \mathrm{~nm}$ is $>71$-fold compared to that in THF, thus validating the AIE attribute of CZ2TPAN. The photograph, inset in Figure 2(b), shows the vivid emission contrast of CZ2TPAN in THF and 10/90 $\mathrm{THF} /$ water, further manifesting the nonluminescent and highly emissive features of the molecularly dissolved species and nanoaggregates, respectively.

Absorption spectrum of CZ2TPAN in 10/90 THF/water mixture is shown in Figure S2, which shows maxima at $303 / 385 \mathrm{~nm}$. Compared with those in THF, both the shorter and longer absorptions are red shifted by 7 and $26 \mathrm{~nm}$, respectively. The result indicates the formation of J-aggregation in the nanosuspensions of CZ2TPAN.

To quantitatively evaluate the AIE effect of CZ2TPAN, quantum efficiencies in the solution $\left(\Phi_{\mathrm{f}, \mathrm{s}}\right)$ and as prepared solid powder $\left(\Phi_{\mathrm{f}, \text { asp }}\right)$ states were determined. While the $\Phi_{\mathrm{f}, \mathrm{s}}$ value in THF is merely $0.12 \%, \Phi_{\mathrm{f} \text {,asp }}$ is significantly boosted to $65.3 \%$, giving an $\operatorname{AIE}$ factor $\left(\alpha_{\mathrm{AIE}}=\Phi_{\mathrm{f}, \text { aps }} / \Phi_{\mathrm{f}, \mathrm{s}}\right)$ as high as 544.
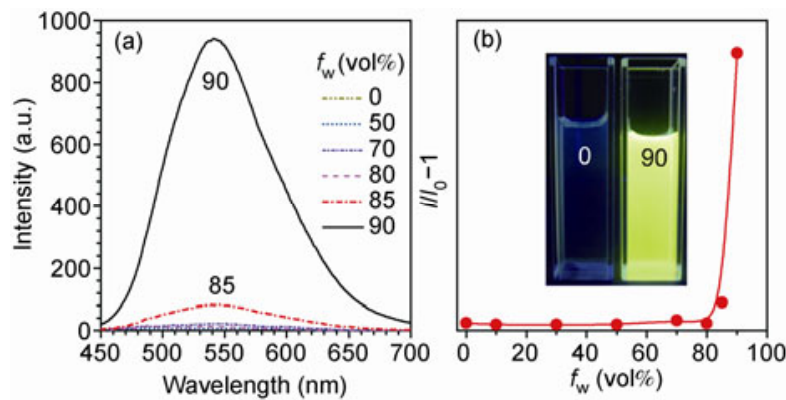

Figure 2 (a) PL spectra of CZ2TPAN in THF and THF/water aqueous mixtures. (b) Plot of PL intensity at $541 \mathrm{~nm} v s$ water fraction $\left(f_{\mathrm{w}}\right)$ for CZ2TPAN. The graph in panel (b) shows CZ2TPAN in THF and 10/90 $\mathrm{THF} / \mathrm{H}_{2} \mathrm{O}$ under UV irradiation. Concentration $=20 \mu \mathrm{mol} / \mathrm{L}$; excitation wavelength $=410 \mathrm{~nm}$. 
Time resolved fluorescence spectra of CZ2TPAN were also measured in solution (THF) and as prepared solid powder states for better understanding of the decay dynamics of the excited states. As summarized Table 1, two relaxation channels, one fast $\left(\tau_{1}, 0.43 \mathrm{~ns}, 61.23 \%\right)$ and the other slow $\left(\tau_{2}, 2.19 \mathrm{~ns}, 38.77 \%\right)$ pathways, for CZ2TPAN in THF are obtained, giving a mean lifetime $(\langle\tau\rangle)$ of $1.11 \mathrm{~ns}$. While there are two emitting species present in THF, only one with a much longer $\langle\tau\rangle$ of $2.83 \mathrm{~ns}$ is found in the solid, which implies that the fast decay channel is closed and the slow channel is populated upon aggregation. Meanwhile, much longer lifetime of CZ2TPAN in the solid state might be ascribed to its more effective light emission.

High solid-state efficiency and AIE characteristic render CZ2TPAN promising candidates as sensors. When dotted (from its DCM solution) on a thin-layer chromatography (TLC) plate, letters of "1988" generate bright yellow light under 365-nm UV light (Figure 3(a)). However, such emission disappears in an atmosphere of chloroform vapor (Figure 3(b)), suggesting the sensory capacity of CZ2TPAN towards volatile organic compounds (VOC). Such emission on-off switching is reversible, which is directly associated to the restriction and activation of intramolecular rotations of CZ2TPAN without and in chloroform vapor, respectively.

Both AIE-active compounds and D-A structured luminogens are promising mechanochromic materials [12]. Therefore, we checked whether CZ2TPAN is mechanochromic. While the as prepared green solid powders of CZ2TPAN emit powerful green lights with maximum at $504 \mathrm{~nm}$, their ground yellow counterparts generate a strong yellow emission centered at $545 \mathrm{~nm}$ (Figure 4(a) and (b)), thus indicating its mechanochromic nature. Meanwhile, upon exposure to chloroform vapor for half an hour, yellow

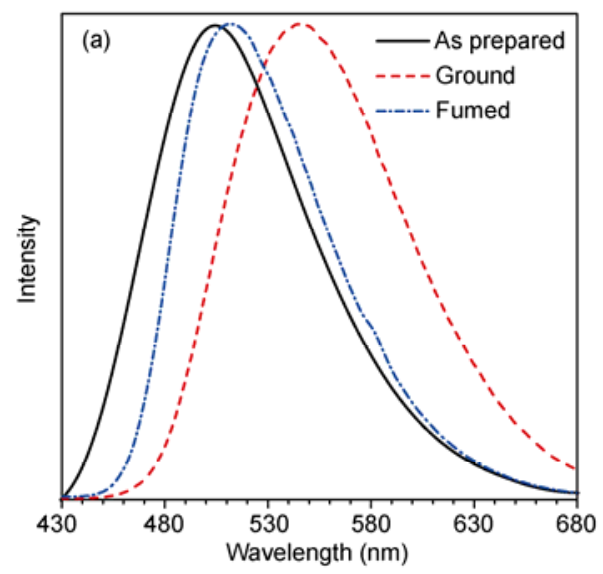

(b)

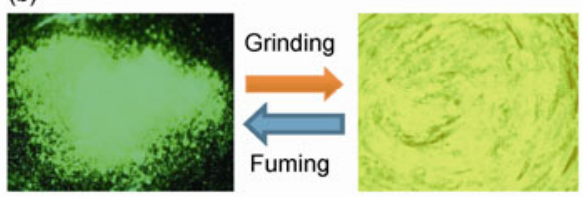

Table 1 PL lifetime for CZ2TPAN in THF solution and as prepared solid powder states

\begin{tabular}{lccccc}
\hline Samples & $A_{1}(\%)^{\mathrm{a})}$ & $A_{2}(\%)^{\mathrm{a})}$ & $\tau_{1}(\mathrm{~ns})^{\mathrm{b})}$ & $\tau_{2}(\mathrm{~ns})^{\mathrm{b})}$ & $\langle\tau\rangle(\mathrm{ns})^{\mathrm{c})}$ \\
\hline Solution & 61.23 & 38.77 & 0.43 & 2.19 & 1.11 \\
Solid & & & & 2.83 & 2.83 \\
\hline
\end{tabular}

a) Fractional contribution. b) Fluorescence lifetime. c) Weighted mean lifetime.
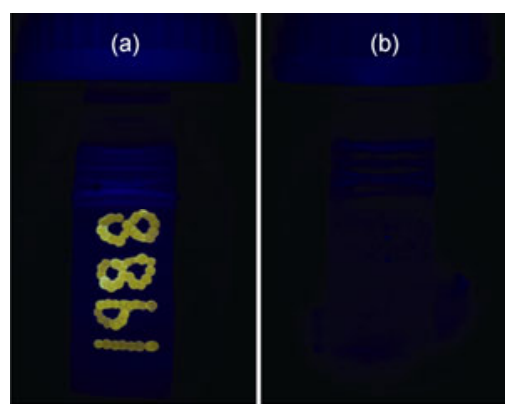

Figure 3 On/off fluorescence switching of CZ2TPAN on the TLC plate without vapor (a) and in chloroform vapor (b) under 365-nm UV light illumination.

powders are restored to green solids again, which emits bright green light at $510 \mathrm{~nm}$ (Figure 4(a)).

To get more insights into the mechanism of the mechanochromism, X-ray diffraction (XRD) analysis was performed. As shown in Figure 4(c), the XRD profile of the as prepared solid clearly displays intensive and sharp diffraction peaks, reflecting a well-ordered microcrystalline structure. However, the diffractogram of the ground solid shows only a weak and broad diffusion halo, suggesting its disordered amorphous stacking. After being fumed with solvent vapor, noticeable diffractions resemble to those of the as prepared solid emerge out, indicating the recovery of

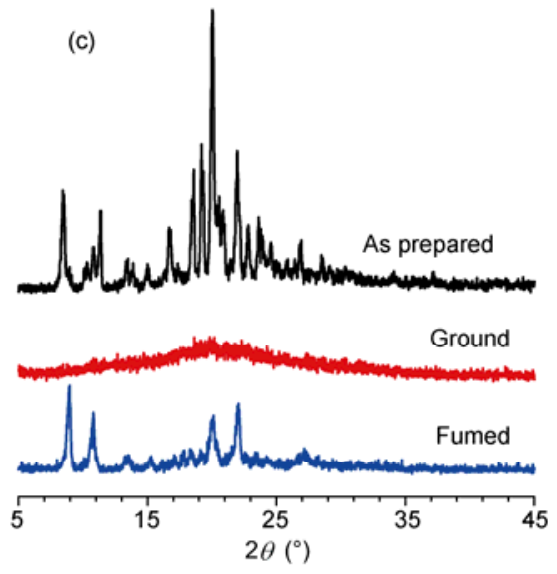

(d)

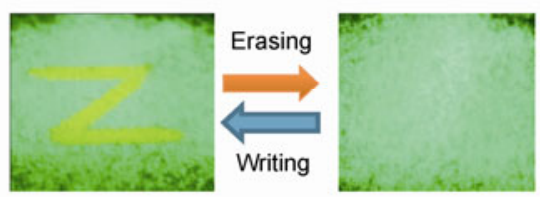

Figure 4 (a) Normalized PL spectra, (b) photographs under 365-nm UV light and (c) XRD patterns of CZ2TPAN at different states as indicated. (d) Writing and erasing of letter " $Z$ " on the filter paper with sprayed CZ2TPAN under UV light. 
ordered crystalline lattice. According to these results, it is reasonable to ascribe the mechanochromism as a reversible phase transition process between order crystalline and disordered amorphous states. The red shifted emission upon mechanical stimuli should be attributed to the planarization of highly twisted conformations of CZ2TPAN, which induces much longer effective conjugation length and thus much redder emission.

Switchable mechanochromic luminescence with a remarkable shift of $41 \mathrm{~nm}$ endows high efficiency CZ2TPAN solid applicable in varying fields, for example in the rewritable optical storage. As depicted in Figure 4(d), after being simply pressed by a glass rod on a filter paper with sprayed CZ2TPAN, the yellow letter "Z" can be clearly distinguished from the green background under 365-nm UV light irradiation. Upon fuming with chloroform vapor for 1 min, "Z" disappears. Such switching can be repeated many times without fatigue because there is no chemical structure destruction for CZ2TPAN in the pressing and fuming processes.

In summary, a novel D-A structured compound CZ2TPAN showing typical AIE and ICT characteristics with high solid-state efficiency of $65.3 \%$ has been successfully obtained. Luminescent colors of solid CZ2TPAN can be smartly switched by various environmental stimuli, such as mechanical force and organic vapor. Pressing and fuming treatments can effectively induce distinct fluorescence color changes between green and yellow owing to the crystalline-amorphous phase transformations. These intriguing properties enable CZ2TPAN for a variety of promising applications in chemical sensors, environment protection, rewritable record media, and light-emitting devices.

This work was supported by the National Natural Science Foundation of China (21104044), the National Basic Research Program of China (2013CB834704), and the Ph.D. Programs Foundation of Ministry of Education of China (20110073120040). YUAN WangZhang thanks the Start-up Foundation for New Faculties of Shanghai Jiao Tong University.

1 Yoshimitsu S, Toshiki M, Isao Y, et al. J Am Chem Soc, 2007, 129: $1520-1521$

2 Dou C D, Han L, Zhao S, et al. J Phys Chem Lett, 2011, 2: 666-670

3 Luo X L, Li J N, Li C H, et al. Adv Mater, 2011, 23: 3261-3265

4 Zhang X Q, Chi Z G, Li H Y, et al. Chem Asian J, 2011, 6: 808-811

5 Andrea P, Flavia D C, Francesca S, et al. J Mater Chem, 2007, 17: 783-790

6 Dong Y Q, Jacky W Y Lam, Qin A J, et al. Appl Phys Lett, 2007, 91 : 011111

7 Akihiro K, Takashi Y, Kentaro Y, et al. Nat Mater, 2005, 4: 546-549

8 Shuzo H, Toshiyuki W. Adv Mater, 2006, 18: 2725-2729

9 Zhang H Y, Zhang Z L, Ye K Q, et al. Adv Mater, 2006, 18: 2369-2372

10 Dong Y J, Xu B, Zhang J B, et al. Angew Chem Int Ed, 2012, 51: 10782-10785

11 Birks J B. Photophysics of Aromatic Molecules. London: Wiley, 1970

12 Chi Z G, Zhang X Q, Xu B J, et al. Chem Soc Rew, 2012, 41: 3878-3896

13 Luo J D, Xie Z L, Jacky W Y Lam, et al. Chem Commun, 2001, 18: $1740-1741$

14 Zeng Q, Li Z, Dong Y Q, et al. Chem Commun, 2007, 1: 70-72

15 Hong Y N, Jacky W Y Lam. Chem Commun, 2009, 29: 4332-4353

16 Sun F, Zhang G X, Zhang D Q. Chin Sci Bull, 2012, 57: 4284-4288

17 Li Z. Chin Sci Bull, 2010, 55: 2924-2925

18 Yuan W Z, Gong Y Y, Chen S M, et al. Chem Mater, 2012, 24: $1518-1528$

Open Access This article is distributed under the terms of the Creative Commons Attribution License which permits any use, distribution, and reproduction in any medium, provided the original author(s) and source are credited.

\section{Supporting Information}

Figure S1 APCI mass spectrum of CZ2TPAN.

Figure S2 Normalized absorption spectra of CZ2TPAN in THF and 10/90 THF/ $\mathrm{H}_{2} \mathrm{O}$ mixture. Concentration $=20 \mu \mathrm{mol} / \mathrm{L}$.

The supporting information is available online at csb.scichina.com and www.springerlink.com. The supporting materials are published as submitted, without typesetting or editing. The responsibility for scientific accuracy and content remains entirely with the authors. 